\title{
BMJ Open Description and assessment of trustability of motives for self-exclusion reported by online poker gamblers in a cohort using account-based gambling data
}

\author{
Amandine Luquiens, ${ }^{1,2}$ Delphine Vendryes, ${ }^{2}$ Henri-Jean Aubin, ${ }^{1}$ Amine Benyamina, ${ }^{1}$ \\ Stéphane Gaiffas, ${ }^{2,3}$ Emmanuel Bacry ${ }^{2,4}$
}

To cite: Luquiens A,

Vendryes D, Aubin H-J, et al. Description and assessment of trustability of motives for self-exclusion reported by online poker gamblers in a cohort using account-based gambling data. BMJ Open 2018;8:e022541. doi:10.1136/ bmjopen-2018-022541

\section{- Prepublication history for} this paper is available online. To view these files, please visit the journal online (http://dx.doi. org/10.1136/bmjopen-2018022541).

Received 23 February 2018 Revised 23 October 2018 Accepted 7 November 2018

D) Check for updates

(c) Author(s) (or their employer(s)) 2018. Re-use permitted under CC BY-NC. No commercial re-use. See rights and permissions. Published by BMJ.

${ }^{1}$ Hôpital Paul Brousse, APHP, Université Paris-Saclay, Université Paris-Sud, UVSQ, CESP, INSERM, Villejuif, France ${ }^{2}$ CMAP, Ecole Polytechnique, Palaiseau, lle-de-France, France ${ }^{3}$ LPSM UMR 8001, Université Paris Diderot, Paris, France ${ }^{4}$ CEREMADE (CNRS UMR 7534), Université Paris-Dauphine-PSL, Paris, France

Correspondence to Dr Amandine Luquiens; amandineluquiens@gmail.com

\section{ABSTRACT}

Objective Self-exclusion is one of the main responsible gambling tools. The aim of this study was to assess the reliability of self-exclusion motives in self-reports to the gambling service provider.

Settings This is a retrospective cohort using prospective account-based gambling data obtained from a poker gambling provider.

Participants 0 ver a period of 7 years we included all poker gamblers self-excluding for the first time, and reporting a motive for their self-exclusion $(n=1996)$. We explored two groups: self-excluders who self-reported a motive related to addiction and those who reported a commercial motive.

Results No between-group adjusted difference was found on gambling summary variables. Sessions in the two groups were poorly discriminated one from another on four different machine-learning models. More than two-thirds of the gamblers resumed poker gambling after a first self-exclusion $(n=1368)$, half of them within the first month. No between-group difference was found for the course of gambling after the first self-exclusion. $60.1 \%$ of first-time self-excluders self-excluded again $(n=822)$. Losses in the previous month were greater before second self-exclusions than before the first.

Conclusions Reported motives for self-exclusion appear non-informative, and could be misleading. Multiple selfexclusions seem to be more the rule than the exception. The process of self-exclusion should therefore be optimised from the first occurrence to protect heavy gamblers.

\section{INTRODUCTION}

Gambling disorder is linked to considerable social and public health damage, ${ }^{1}$ but characterised by a substantial treatment gap and delay between the onset of the disorder and access to care. 'Responsible gambling' tools do exist, but have mostly been developed empirically, relying on a low level of evidence. ${ }^{23}$ Self-exclusion is one of the main tools for responsible gambling. Gamblers can
Strengths and limitations of this study

- This is the first study prospectively documenting self-reported motives for self-exclusion.

- Our study systematically included self-excluders who reported a motive during the usual self-exclusion process, limiting self-selection bias.

- No information on offline gambling and gambling on other websites was available.

- No comparison group was included in the analysis. The efficacy of self-exclusion should be assessed in a future study comparing self-excluders with matched non-self-excluders.

apply for offline self-exclusion for one particular website, or for both online websites and offline venues (ie, casino venues). However, little is known about the characteristics of those who choose to apply for this measure, about their motivation to do so, or its effectiveness. Most studies in the field have recruited self-selected self-excluders. Gambling characteristics are then collected retrospectively, whether online, ${ }^{4}$ or offline, including motives for self-exclusion, that is, role of problem gambling. ${ }^{5}$ As a consequence, recall and recruitment biases have been major issues. Some studies on online gamblers have suggested a surprisingly moderate percentage of problem gamblers $(68 \%),{ }^{6}$ contested by other surveys reporting up to $95 \%$ problem gamblers among offline self-excluders. ${ }^{7}$ Some studies have pointed to heterogeneity among self-excluders. ${ }^{6}$

To date, no prospective information on motives for self-exclusion has been made available. However, two studies on online self-exclusion have used prospectively recorded account-based gambling data. ${ }^{8}$ Accountbased gambling data offer the opportunity to 
report gambling behaviours on a website objectively and with no recall bias. Data of this type have been reported in other contexts to describe problem gambling behaviours. ${ }^{10}{ }^{11}$ Dragicevic et al showed that self-excluders $(n=347)$ on average sustained higher losses and presented riskier behaviours than controls. However, for technical reasons, these authors chose to include only mono-self-excluders and self-excluders for at least 30 days. ${ }^{8}$ They found a high rate of very quick self-excluders (25\% within a day). On the other hand, Xuan and Shaffer demonstrated a change in gambling behaviour in the days prior to self-exclusion. ${ }^{9}$

Unfortunately, no follow-up data were available in these studies. Effectiveness analyses have been reported among pathological gamblers in land-based gambling venues and remain scarce and generally concern self-selected samples. To date, despite methodological issues and weak evidence, self-exclusion has often been presented as a therapeutic tool in itself. If previous studies demonstrated significant changes among pathological gamblers in land-based gambling venues from before exclusion to after exclusion, no comparative data are available to state on the efficacy of self-exclusion. ${ }^{12}$ Poker gamblers could be a particularly interesting population in which to explore self-reported motives for self-exclusion, as they present particularities in terms of feelings of belonging to a gambling community and frequently claim a passion for the activity rather than problematic behaviour. ${ }^{13}$ However, it has been shown that only harmonious passion is unrelated to problem gambling, unlike obsessive passion, among poker gamblers. ${ }^{14}$ Poker is a game that implies skills. The perception of one's own skills could be increased by marketing slogans, and also by the gambling media: it has been demonstrated that online gamblers have a more marked perception of possessing particular skills compared with offline gamblers, despite the fact that they do not in fact possess superior ability. ${ }^{15}$ Internet poker gamblers could have a false perception of their own behaviour, or deliberately report a misleading motive to the gambling service provider as a result of the specificities of the game and its societal representations. ${ }^{16}$ The prevalence of problem gamblers among online poker gamblers is well documented and very high $(17 \%) .{ }^{11}$ Damage related to gambling is known to impact many areas in the gambler's life and his/her relatives. ${ }^{1}$ An increase in online gambling and an increasing level of problem gambling since the regulation of online gambling in France ${ }^{17}$ justify the need for documentation on the usefulness of existing responsible gambling tools such as self-exclusion.

The aim of this study was to ${ }^{1}$ describe a sample of poker gamblers who self-excluded, systematically included over 7 years, before and after the first self-exclusion event, ${ }^{2}$ to assess the trustworthiness of self-reported motives for self-exclusion.

\section{METHODS}

\section{Population}

All poker gamblers who self-excluded at least once from the Winamax poker gambling website since its launch in
June 2010 up to October 2016, and who provided reasons for this first self-exclusion, were systematically included. Motives for self-exclusion are routinely prospectively collected by the gambling service provider: in the course of the self-exclusion process on the website, gamblers can motivate their choice to self-exclude with one of the following proposed reasons: (1) internalised motives related to addiction, and (2) externalised motives related to commercial considerations. (1) Internalised motives related to addiction motives mentioning the person's relationship with gambling, for example, negative consequences of gambling, loss of control and need to change gambling practice to protect oneself: 'You think this activity costs you too much money', 'You have trouble being in control of yourself when you play poker', 'You do not want to play poker online any more'. These three motives can be linked to motivations to stop problem gambling, according to the self-determination theory. ${ }^{18}$ In particular, and to illustrate this proximity, the Treatment Self-Regulation Scale, based on the self-determination theory, and modified to include questions relating to gambling, ${ }^{19}$ collects responses to questions 'The reason I would stop gambling permanently or continue not to gamble heavily is....' Constructs identified as part of this scale include 'identified motivation' (four items; eg, 'Because I personally believe it is the best thing for my health'); 'integrated motivation' (two items; eg, 'Because it is consistent with my life goals'); 'external motivation' (four items; eg, 'Because others will be upset with me if I gamble'); 'introjected motivation' (three items; eg, 'Because I would feel bad about myself if I gambled'); and 'amotivation' (three items; eg, 'I don't really know why'). ${ }^{18}$ The motives proposed here, 'You think this activity costs you too much money' and 'You have trouble being in control of yourself when you play poker', appear to reflect 'identified motivation' to quit gambling. The third motive, 'You do not want to play online poker any more', appears to reflect a strong willpower to change, common in addiction, ${ }^{20}$ with the use of the verb 'want' in the wording of the proposed motive, but with no wish to give a reason on the website or a difficulty in expressing internal, external or introjected motivations. Moreover, the statement 'I don't want to gamble any more' is very close to the only requirement for Gamblers Anonymous membership from the 12 traditions, 'a desire to stop'. ${ }^{21}$ To support our view that this motive is related to addiction, we conducted a Google search with the wording of this motive, 'I don't want to gamble any more', yielding 22 results. Twenty of the 22 results referred directly to addiction and problem gambling. Among the two remaining results, one was outside the scope of this research, as it was a metaphorical use of the word 'gamble', referring to taking a chance, and the other came with no additional contextual information, as it was the only sentence in a video. These findings support our design, which considers the statement 'I don't want to gamble any more' as related to addiction. (2) Externalised motives related to commercial considerations were motives with 
no mention of the person's gambling behaviour and explicitly mentioning the commercial offer: 'You do not like the software', 'You play on a competing website', 'The loyalty program (Status, miles, bonus) does not suit you', 'The tournament offer does not suit you', 'You are not satisfied with customer support', 'You have encountered security problems'. Self-excluders did not have to report a motive, and they could either skip the question, report no motive or choose the 'other motive' option. We did not include gamblers who chose either of these two options. Self-exclusion duration is fixed by the player from 1 day up to a maximum of 3 years. At the end of the self-exclusion period the gamblers are notified by email by the provider, and they are then allowed to gamble again on the platform without any additional procedure. At no point during the self-exclusion process is guidance or any kind of help offered. Self-exclusion prevents the gambler from any kind of gambling activity on the website during the chosen period of time. Self-prohibition is a heavier voluntary 3-year process that prevents the gambler from any kind of gambling activity online or on casino venues; the motive is necessarily addiction in France, and has been classified in this study as self-exclusion for a motive relating to addiction. A detailed flow chart is provided in figure 1.

\section{Patient and public involvement \\ Patient or public were not involved.}

\section{Measures}

We collected data retrospectively from different prospective databases systematically recorded by the gambling service provider: (A) Gambler data: self-excluders' basic demographics (gender, age, date since opening of the account), characteristics of self-exclusions (date, self-reported motive) and gambling summary variables in the month prior to self-exclusion (details of deposits, and basic betting activity in the month) (ie, the amounts bet). We chose to explore the month before each self-exclusion for several reasons. First, the short period before self-exclusion has been reported to be characterised by an increase in gambling involvement in cases where self-exclusion is related to gambling problems. ${ }^{9}$ These authors used a 23-day period, considered to be relevant for most of their sample. Other studies chose to explore a 1 month period. ${ }^{8}$ In a previous study on poker gamblers we examined account-based gambling data in the previous month and were able to demonstrate that it was predictive of problem gambling as assessed with a classic screening tool. ${ }^{11}$ Most employed people in France receive their income once a month. It is therefore important to capture at least 1 month to avoid any artificially enhanced gambling activity resulting from a possible effect following receipt of income. (B) Session characteristics: starting date, end date, duration. Sessions were defined as gambling with no time span $>10$ min with no action. (C) Cash game and tournament gambling data at table level for players with real money. Winnings were computed from table data (cash game winnings+prize amount for tournaments-buy-in-plus-rake for tournaments). Data were available from the launch of the website in June 2010 up to February 2017 (ie, at least 4 months after the last self-exclusion included).

\section{Analysis}

We present data for the whole sample and for two groups: those with a first self-exclusion for a motive related to addiction (group 1) and those with a first self-exclusion for commercial motives (group 2). Our hypothesis was that group 1 would demonstrate more intense gambling behaviour before the first self-exclusion and a more deleterious course than group 2. We present results on three levels:

1. Gambling summary variables for the month prior to the first self-exclusion, and to the second self-exclusion if there was a second one. We described and compared sociodemographics and gambling summary variables in the month before the first self-exclusion between subjects who self-excluded for addiction and those who self-excluded for commercial reasons, by bivariate and multivariate analyses (logistic regression). We also described sociodemographics and gambling summary variables in the month before the second self-exclusion.

2. Session characteristics for the month preceding self-exclusion. We described and compared session characteristics in the month before the first self-exclusion between the subjects who self-excluded for addiction and those who self-excluded for commercial reasons, using four machine-learning classification models (logistic regression, K-nearest neighbour (KNN) classifier, decision tree classifier and a random forest classifier). We performed a cross-validation and computed different parameters (accuracy, receiver operating characteristic-area under the ROC curve (ROC-AUC), recall score). All the above analyses were performed using Python with the open source scikit-learn library. ${ }^{22}$

3. Return to gambling on the website. We ran a mixed model for repeat measurement analyses, comparing the course of the winnings over 4 months after the return to gambling between the two groups, testing an interaction between time and group as a fixed effect, and random subject and time effects (R3.4.3 software, lme4 package). For this model, missing data on the variables concerning winnings, meaning no winnings because no gambling with real money over the period, were completed as 0 . The $p$ value was obtained by performing an analysis of variance between this model and the null model.

\section{Secondary analysis}

We performed an additional analysis on our data set, on a subsample excluding gamblers that reported the least explicit motive 'I don't want to gamble any more' $(\mathrm{n}=1694)$ to confirm our design: between-group comparison on summary gambling characteristics in the month 


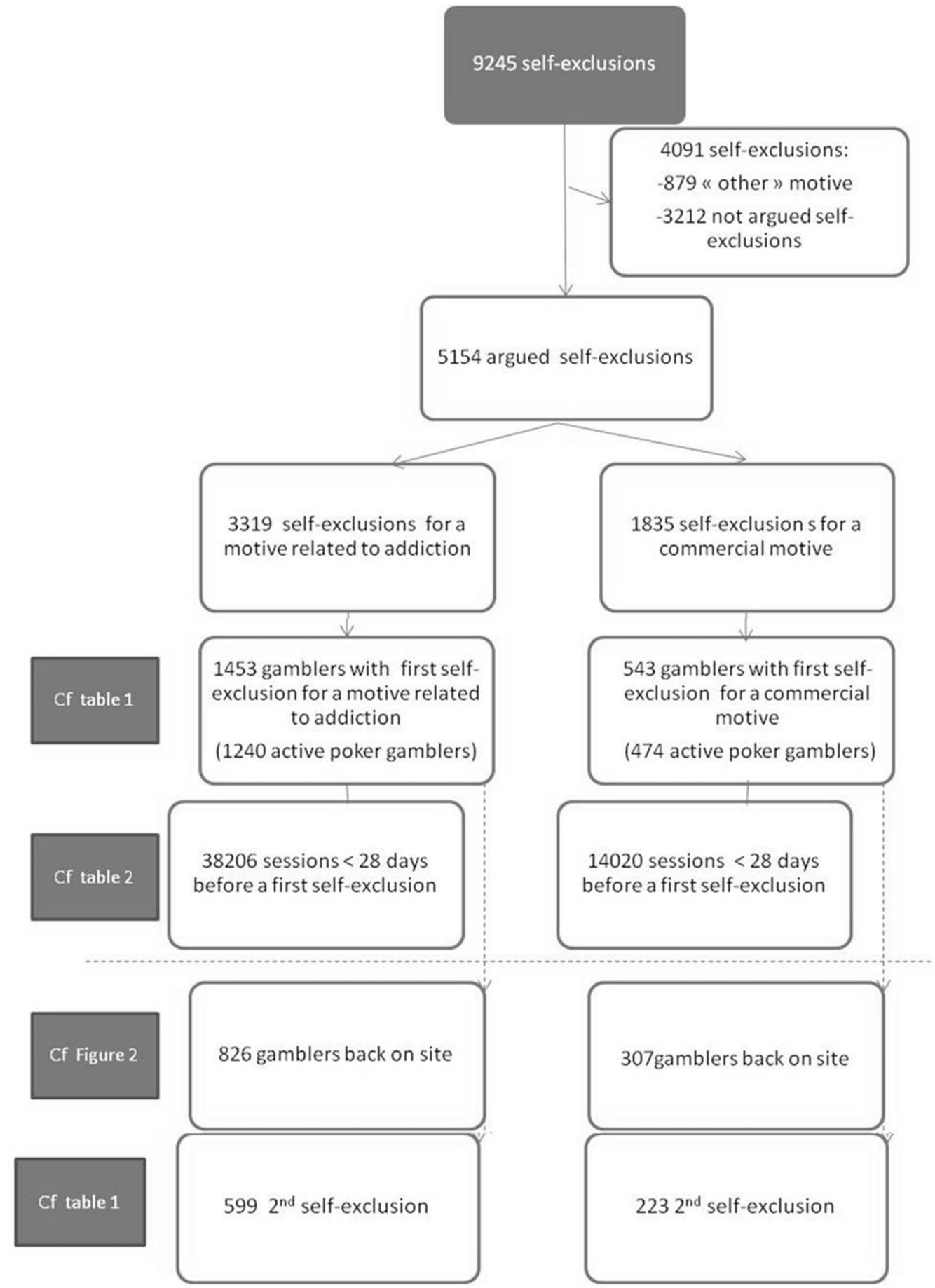

Figure 1 Flow chart.

before self-exclusion and between-group differences for the course of gambling using a mixed model.

\section{RESULTS}

First self-exclusion and between-group motive differences

In 6 years and 4 months, 1203558 gamblers opened an account on the gambling website, and 4887 gamblers self-excluded at least once ( $0.4 \%$ of gamblers). Our analysis included the 1996 different gamblers who gave a motive for their first self-exclusion, including 1714 poker gamblers with real-money active players in the month before the first self-exclusion. The other 282 players were poker gamblers who had an active account with real money activated on the platform at the time of self-exclusion, but who did not play poker with real money at all in the previous month (12.7\% of group $2(n=69)$ and $14.7 \%$ of group $1 \quad(n=213), X^{2}$ test $\left.p>0.05\right)$. They may have played with fake money, but we had no access to this activity. More than half of them had no sports betting activity ( $\mathrm{n}=138)$. Addictive behaviour-related motives 
Table 1 Demographic and gambling behaviour characteristics before first and second self-exclusions

The month before a first self-exclusion

$\begin{array}{ll}\text { All gamblers with } & \begin{array}{l}\text { All gamblers with a } \\ \text { first exclusion for }\end{array} \\ \text { motivated first } & \text { a motive related to } \\ \text { exclusions }(n=1996) & \text { addiction }(n=1453)\end{array}$

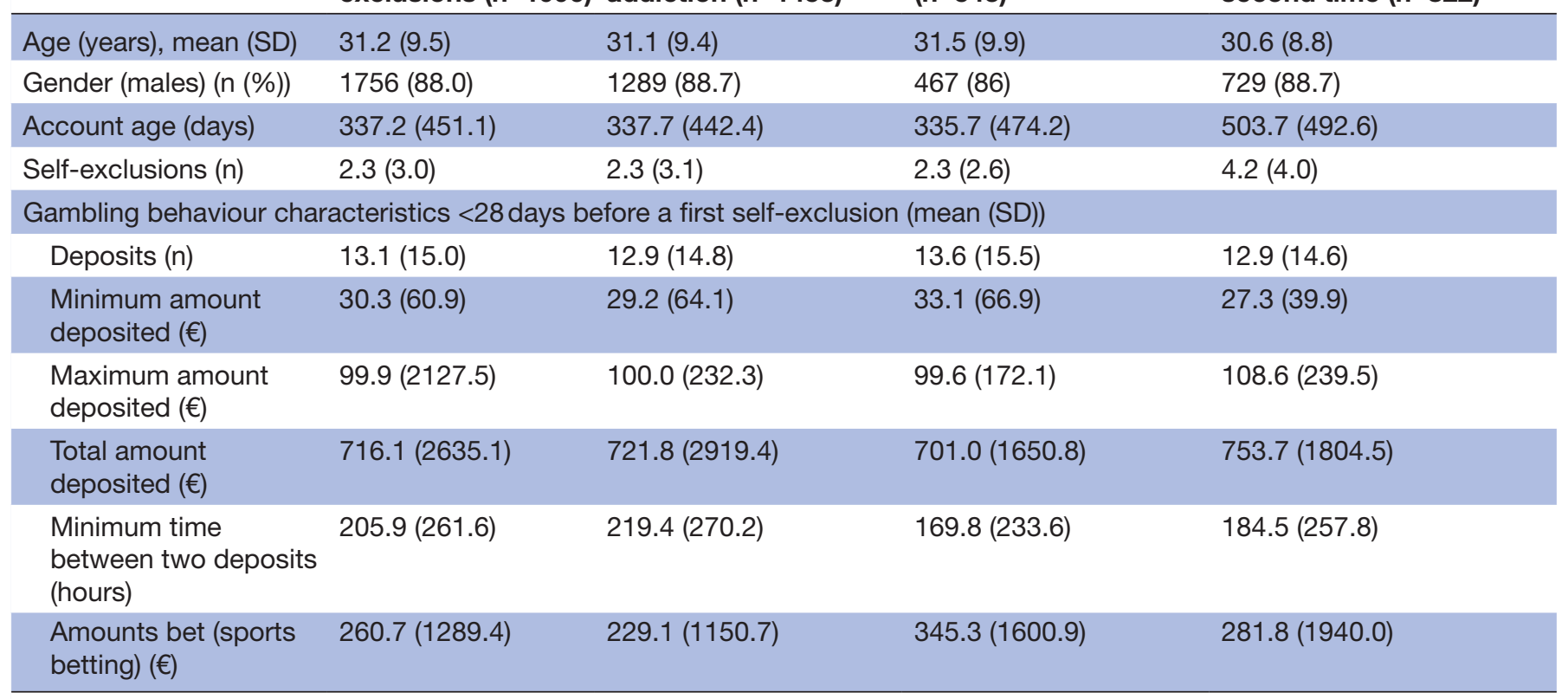

No adjusted significant difference between groups in the logistic regression.

were nearly three times more frequent than commercial motives (1453 vs 543). The mean age was 31 years and most of the gamblers were male $(88 \%)$. The first self-exclusion occurred on average in the last trimester of the first year after opening an account (mean=337 days \pm SD). Gamblers made on average 13 deposits in a month, with a mean range of $€ 30-€ 100$ per deposit. The average total deposit was $€ 716$. Compulsivity, which was measured by the minimum time between two deposits, was the reverse of what was expected, that is, it was shorter among self-excluders for commercial motives than among those for addictive motives (9 vs 7 days on average). Sport betting was documented using the betting amount on the website, and was $€ 260$ a month on average. Gamblers' detailed characteristics and gambling summary characteristics are presented in table 1 . Gamblers had an average of 30.3 sessions a month $(\mathrm{SD}=28.5)$. Regarding the characteristics of the sessions $(n=52226)$, gamblers gambled mostly during the day (8-21 hours) and on business days. The duration of the sessions was $77.8 \mathrm{~min}$ on average $(\mathrm{SD}=97.7)$. Financial losses per cash game session were $€ 36.7$ (SD=196.3) on average, the players' buy-in-plusrake was $€ 49.6$ ( $\mathrm{SD}=181.9)$ and the amount of winnings was $€ 40.4$ (184.4) on average for tournaments. Detailed characteristics of the sessions are presented in table 2.

In the analysis of the month before the first exclusion, several between-group differences were found in bivariate analyses on sociodemographics and month-summary variables: the minimum time between two deposits (371076.5, $\mathrm{p}=0.020$ ), the minimum and maximum amounts per deposit (respectively 361772.0, $\mathrm{p}=0.002$ and 374540.5, $\mathrm{p}=0.040)$, the total deposit $(371273.0, \mathrm{p}=0.021)$, gender (383801.5, $\mathrm{p}=0.049$ ) and the amount bet (365623.0, $\mathrm{p}=0.000)$. Mean differences were small, except for the amount bet and the minimum time between two deposits. However, no significant between-group adjusted difference was found. We found no acceptable classifier to discriminate sessions between the two groups using four machine-learning classification models (logistic regression, KNN classifier, decision tree classifier and a random forest classifier). The random forest model gave the best performance: accuracy was 0.72 with a very low recall score (0.16 for commercial reasons, 0.89 for addictive reasons), and the AUC was 0.57 .

\section{Follow-up}

More than two-thirds $(68.5 \%, \mathrm{n}=1368)$ of the gamblers were back gambling on the website after their first exclusion, the mean time to return to poker gambling was 65 days (median 30 days, $25 \% 13$ days, $75 \% 87$ days). Losses decreased between the month before the first self-exclusion and the first month after returning to gambling for those back on the website. No between-group difference was found for the course of winnings over the period explored $(p=0.64)$. The number of gamblers who continued gambling on the website decreased over time after the return, partly because of subsequent self-exclusions. On average, gamblers self-excluded twice. 
Table 2 Characteristics of gambling sessions according to self-exclusion motive in the month before first self-exclusion

All sessions

aggregated by

gambler,

$<28$ days before a

first self-exclusion

All gamblers with All gamblers with

a first exclusion first exclusion

for commercial

(mean (SD))

motives $(n=38206)$ reason $(n=14020)$

Time period (score)

$\begin{array}{lcc}\text { Day } & 0.7(0.5) & 0.7(0.5) \\ \text { Evening } & 0.2(0.4) & 0.2(0.4) \\ \text { Night } & 0.1(0.3) & 0.1(0.3) \\ \begin{array}{l}\text { Weekend } \\ \text { Length (min) }\end{array} & 0.3(0.4) & 0.3(0.4) \\ \begin{array}{l}\text { Cash game } \\ \begin{array}{l}\text { Maximum } \\ \text { amount played }\end{array}\end{array} & 659.8(96.0) & 80.8(118.2) \\ \begin{array}{l}\text { Total amount } \\ \text { played }\end{array} & 1475.7(7758.5) & 687.7(2189.0) \\ \begin{array}{l}\text { Mean initial_buy- } \\ \text { in per table }\end{array} & 45.0(79.7) & 68.3(201.5)\end{array}$

in per table

\begin{tabular}{|c|c|c|}
\hline $\begin{array}{l}\text { Maximum } \\
\text { frequency of } \\
\text { staked hands }\end{array}$ & $0.9(0.2)$ & $0.9(0.2)$ \\
\hline $\begin{array}{l}\text { Mean frequency } \\
\text { of staked hands }\end{array}$ & $0.8(0.2)$ & $0.8(0.1)$ \\
\hline $\begin{array}{l}\text { Stake level } \\
\text { (amount played/ } \\
\text { played hands } \times \text { big } \\
\text { blind) }\end{array}$ & $9.1(9.3)$ & $9.5(9.6)$ \\
\hline Tables (n) & $3.3(5.3)$ & $2.8(3.5)$ \\
\hline Go fast (score) & $0.0(0.1)$ & $0.0(0.2)$ \\
\hline Buy-ins (n) & $9.1(36.4)$ & $7.5(20.6)$ \\
\hline $\begin{array}{l}\text { Total amount of } \\
\text { buy-ins }\end{array}$ & $254.1(860.0)$ & $542.8(2704.6)$ \\
\hline $\begin{array}{l}\text { Maximum } \\
\text { winnings }\end{array}$ & 19.0 (153.9) & $55.4(425.8)$ \\
\hline Total winnings & $-36.9(250.9)$ & $-36.2(718.8)$ \\
\hline $\begin{array}{l}\text { Multiple devices } \\
\text { used }\end{array}$ & $1.0(0.6)$ & $1.0(0.4)$ \\
\hline
\end{tabular}

\begin{tabular}{lcc}
\hline Tournament & & \\
\hline Tournaments $(\mathrm{n})$ & $5.4(9.1)$ & $5.9(10.3)$ \\
\hline Add-ons $(\mathrm{n})$ & $0.0(0.2)$ & $0.0(0.2)$ \\
\hline Rebuys $(\mathrm{n})$ & $0.1(0.4)$ & $0.1(0.6)$ \\
\hline $\begin{array}{l}\text { Amount of buy- } \\
\text { in-plus-rakes }\end{array}$ & $47.1(163.1)$ & $56.2(224.5)$ \\
$\begin{array}{l}\text { Level of risk } \\
\text { Amount prize }\end{array}$ & $0.6(0.4)$ & $0.6(0.4)$ \\
$\begin{array}{l}\text { Sit-and-go } \\
\text { tournaments per } \\
\text { session }(\mathrm{n})\end{array}$ & $47.9(165.9)$ & $47.0(226.6)$ \\
$\begin{array}{l}\text { Multiple devices } \\
\text { used }\end{array}$ & $1.2(0.6)$ & $4.5(7.3)$ \\
\hline
\end{tabular}

Gamblers who self-excluded a second time self-excluded four times on average. Two or more self-exclusions occurred with 822 gamblers: $41.1 \%$ of all those who self-excluded at least once whether for an addictive or a commercial reason, which corresponds to $60.1 \%$ of those back on gambling after a first exclusion. Only $59.7 \%$ of the gamblers excluded the first and the second time for the same reason (ie, addictive or commercial reason) $(n=491 / 822)$. The mean number of self-exclusions was 2.3 whether for addiction or for commercial reasons (SD respectively 3.1 and 2.6). Second self-exclusions occurred within 3 months of the first self-exclusion for half of the gamblers (median 94 days, mean 161 days, SD 191). The mean total deposit before the second exclusion was higher than before the first self-exclusion $(€ 753.7(1804.5)$ vs $€ 716.1$ (2635.1) respectively) (table 1 ). However, in an analysis of only the 822 gamblers who self-excluded at least twice, it is worth noting that the total deposit before the first self-exclusion was larger than with gamblers who self-excluded only once, and that there was no increase in total deposits between the first and second exclusions $(€ 883.1$ (1824.6) vs $€ 753.7(1804.5))$.

\section{Secondary analysis}

The additional analysis on the subsample excluding gamblers that reported the least explicit motive 'I don't want to gamble any more' $(\mathrm{n}=194)$ showed similar results: there was no significant between-group difference on summary gambling characteristics in the month before self-exclusion (logistic regression, all $\mathrm{p}>0.05$ ), and no between-group difference for the course of gambling using a mixed model $(\mathrm{p}=0.06331)$.

\section{DISCUSSION}

Our study presents account-based data on gambling characteristics and first self-exclusion motives of 1996 poker gamblers. It is the largest sample of self-excluders ever reported. The inclusion of self-excluders was systematic over 7 years, and motives were collected prospectively, avoiding any recruitment or recall bias. Follow-up was documented objectively and was studied up to the second month after return to the website. We demonstrated that self-excluders were very heavy gamblers, that self-exclusion was mostly a non-unique event and that its protective effect seemed temporary, suggesting a deterioration in practice within 2 months for those returning to the website. Motives for self-exclusion self-reported to the gambling service providers did not seem very relevant.

Both groups were very heavy gamblers in terms of financial outlay ( $€ 716$ deposited in the month on average). The total deposit in a month was on average much higher than the total deposit found in a population of problem gamblers on the same website but included in another study (€248 on average among poker gamblers, with Canadian Problem Gambling Index score $\geq 5) .{ }^{11}$ This suggests that self-excluders could be seen as a population with severe gambling problems. Our population 
presented a mean gambling time of 40 hours in the month, which far exceeds the time involvement reported in Dragicevic's study among self-selected self-excluders (total gambling time per month 19.5 hours). This could be explained by more intense gambling practice, but also at least partly by the fact that our population was made up of poker gamblers, whereas Dragicevic included a mixed population of poker and casino gamblers. Our study with its systematic inclusion supports Ladouceur's hypothesis that all self-excluders are problem gamblers, in contrast with some previous studies involving self-selected subjects. In our study, self-reported motives for self-exclusion seem inconsistent, unreliable and irrelevant. Some gamblers who self-excluded for commercial reasons could indeed be problem gamblers who did not report it as such for different reasons. They could either have had a misperception of their own behaviour or deliberately reported a misleading motive to the gambling service provider. The gambling service provider is probably not the most appropriate party to ask for protection. The motives self-reported to a gambling service provider could be misleading for documenting the underpinnings of self-exclusion for the regulatory authorities. The self-excluders were very heavy gamblers in both motive groups. Gambling service providers should not use these motives to minimise harm assessment related to the gambling offer.

A minority of gamblers did not gamble again on the website after a first self-exclusion. For them, self-exclusion could have been efficient in avoiding excessive gambling in the long term. They appear to be less excessive gamblers than other self-excluders. However, this finding is to take with caution as we have no information on their gambling on other platforms or land-based venues. Self-excluders who returned to gambling presented a decreased investment in the first month back, but nearly two-thirds self-excluded again at least once. Self-exclusion was barely a unique episode, especially among gamblers back on gambling. The gamblers seemed particularly vulnerable to excessive gambling after a first self-exclusion. The protective effect of one self-exclusion on excessive gambling could be limited in time. It is possible that self-exclusion as a cool-off period only has a temporary effect. Our data support Gainsbury's recommendation to propose outside the gaming environment a ban extension for a renewed time period in order to avoid triggering gambling behaviour. ${ }^{23}$ This suggests a poor therapeutic effect of an isolated, single self-exclusion. However, it is worth noting that the self-exclusion process differs between countries, from no guidance at all to professional health counselling in addition to the measure itself. ${ }^{23-25}$ In France, no guidance is provided with self-exclusion. Gamblers, who have reached the milestone of identifying themselves as excessive gamblers and have applied for self-exclusion, should be better guided from their very first self-exclusion. They could be offered referral for relapse prevention counselling. A model of this sort could seem intrusive, but identifying problem gamblers in different venues and orienting them towards care is already routine in some countries, as in casino venues in Switzerland. ${ }^{26}$ Initial face-to-face meeting with an addiction specialist and even a final mandatory meeting have been implemented and assessed in offline venues, and have been shown not to compromise the process in Canada. ${ }^{27}$ The online process should not be a barrier to offering help during the self-exclusion process. No face-to-face interventions have been offered for online problem gambling or shown to be well received with high inclusion rates among individuals who were not initially treatment seekers; these interventions could be an option, including normative feedback. ${ }^{28}$ In addition, our findings support previous results reporting that a very small minority of problem gamblers self-exclude. ${ }^{29}$ The promotion of self-exclusion also differs between countries. ${ }^{23}$ Self-exclusion could be actively proposed for excessive gamblers screened online.

\section{Limitations}

As mentioned above, in France, no guidance at all is provided to accompany self-exclusion: our follow-up data concern only the self-exclusion measure and the 4 months after a return to the website. Gamblers who self-excluded could however benefit from external assistance, and we have no information on this score. Further to this, no information on gambling offline or gambling on other websites was available. Mixed gambling activity was explored through sports betting activity, but other forms of gambling were not documented. However, while the amounts involved could be underestimated, we consider that the dynamic of losses on the website is indeed informative on the control a person has over gambling activities. However, the use of a clinical measure to attest problem gambling would definitely have been useful to support our findings, but was not feasible given the design of our study. Our classification of motives into group 1 and group 2 could also be discussed. Despite our precautions to justify our design, we cannot state that the a posteriori classification of the motives, without an explicit 'addiction' or 'commercial' label for the gambler, is correct and explains the absence of between-group differences. These findings among poker gamblers may not be representative of all gamblers, given the well-documented particularities of poker gamblers among gamblers overall. A further study including matched non-self-excluders is warranted to document the course of heavy gambling and the efficacy of self-exclusion.

\section{CONCLUSIONS}

Self-excluders were found to be very heavy gamblers in this very large study with a systematic inclusion process. Our hypothesis that there would be differences between motive groups for self-exclusion was not supported by our analyses. Motives for self-exclusion, self-reported to the gambling service provider, seem non-informative and could be misleading for documenting the underpinnings of self-exclusion for use by the regulatory authorities. More than two-thirds of the gamblers were back 
on gambling after their first exclusion. Self-exclusion was not a single event for a considerable proportion of gamblers. Self-exclusions seem to have had a temporary effect among those back on gambling. First self-exclusions should be accompanied with additional protective measures and treatment referral. A ban extension for a further time period could be offered systematically, outside the gambling environment. The online process should provide an opportunity to rethink self-exclusion and promote this tool.

Acknowledgements We thank Winamax for allowing access to data through a convention allowing free analysis and interpretation by our academic team.

Contributors AL, DV, HJA, AB, SG and EB contributed to drafting the protocol, data analysis, and drafting and reviewing the manuscript writing.

Funding The authors have not declared a specific grant for this research from any funding agency in the public, commercial or not-for-profit sectors.

Competing interests AL has received sponsorship to attend scientific meetings, speaker honoraria and consultancy fees from Lundbeck, Indivior and ARJEL. AB has received sponsorship to attend scientific meetings, speaker honoraria and consultancy fees from Lundbeck, Mylan, Gilead, Jansenn Cilag and Indivior. HJA has received sponsorship to attend scientific meetings, speaker honoraria and consultancy fees from Bioprojet, D\&A Pharma, Ethypharm, Lundbeck, MerckSerono, Novartis and Pfizer. DV, SG and EB have no conflict of interest to report.

Patient consent for publication Not required.

Ethics approval Gamblers were informed and consented to personal and gambling data collection and analysis in general conditions of use, when opening an account on the website. Data collection and analysis by Winamax are registered with 'Comité National Informatique et Libertés' (CNIL) Declaration No 1430126 that allows analysis of the routinely recorded data for public health purposes.

Provenance and peer review Not commissioned; externally peer reviewed. Data sharing statement № additional data available.

Open access This is an open access article distributed in accordance with the Creative Commons Attribution Non Commercial (CC BY-NC 4.0) license, which permits others to distribute, remix, adapt, build upon this work non-commercially, and license their derivative works on different terms, provided the original work is properly cited, appropriate credit is given, any changes made indicated, and the use is non-commercial. See: http://creativecommons.org/licenses/by-nc/4.0/.

\section{REFERENCES}

1. Langham $\mathrm{E}$, Thorne $\mathrm{H}$, Browne $\mathrm{M}$, et al. Understanding gambling related harm: a proposed definition, conceptual framework, and taxonomy of harms. BMC Public Health 2016;16:80.

2. Caillon J, Grall-Bronnec M, Hardouin JB, et al. Online gambling's moderators: how effective? Study protocol for a randomized controlled trial. BMC Public Health 2015;15:519.

3. Ladouceur R, Shaffer P, Blaszczynski A, et al. Responsible gambling: a synthesis of the empirical evidence. Addict Res Theory 2017;25:225-35.

4. Griffiths MD, Wood RT, Parke J. Social responsibility tools in online gambling: a survey of attitudes and behavior among Internet gamblers. Cyberpsychol Behav 2009;12:413-21.

5. Hayer T, Meyer G. Self-exclusion as a harm minimization strategy: evidence for the casino sector from selected European countries. Gambl Stud 2011;27:685-700.
6. Hayer T, Meyer G. Internet self-exclusion: Characteristics of selfexcluded gamblers and preliminary evidence for its effectiveness. Int $\checkmark$ Ment Health Addiction 2011

7. Ladouceur R, Jacques C, Giroux I, et al. Analysis of a casino's selfexclusion program. J Gambl Stud 2000;16:453-60.

8. Dragicevic S, Percy C, Kudic A, et al. A descriptive analysis of demographic and behavioral data from Internet gamblers and those who self-exclude from online gambling platforms. J Gamb/ Stud 2015;31:105-32.

9. Xuan Z, Shaffer H. How do gamblers end gambling: longitudinal analysis of Internet gambling behaviors prior to account closure due to gambling related problems. J Gamb/ Stud 2009;25:239-52.

10. Griffiths $M$, Whitty M. Online behavioural tracking in internet gambling research: Ethical and methodological issues. IJIRE 2010;3:104-17.

11. Luquiens A, Tanguy ML, Benyamina A, et al. Tracking online poker problem gamblers with player account-based gambling data only. Int J Methods Psychiatr Res 2016;25:333-42.

12. Kotter R, Kräplin A, Pittig A, et al. A systematic review of landbased self-exclusion programs: Demographics, gambling behavior, gambling problems, mental symptoms, and mental health. J Gamb/ Stud 2018.

13. Morvannou A, Dufour M, Brunelle N, et al. Passion for poker and the relationship with gambling problems: a cross-sectional study. International Gambling Studies. 17, 2017.

14. Morvannou A, Dufour M, Brunelle N, et al. One-year prospective study on passion and gambling problems in poker players. J Gamb/ Stud 2018;34:379-91.

15. MacKay TL, Bard N, Bowling M, Bowling HDC, et al. Do pokers players know how good they are? Accuracy of poker skill estimation in online and offline players. Comput Human Behav 2014;31:419-24.

16. Barrault S, Untas A, Varescon I. Special features of poker. Int Gamb/ Stud 2014;14:492-504.

17. Costes J, Eroukmanoff V, Richard J, et al. Les jeux d'argent et de hasard en France en 2014, 2015. avril.

18. Ryan RM, Deci EL. Self-determination theory and the facilitation of intrinsic motivation, social development, and well-being american psychologist. 2000;55:68-78.

19. Kushnir V, Godinho A, Hodgins DC, et al. Motivation to quit or reduce gambling: Associations between Self-Determination Theory and the Transtheoretical Model of Change. J Addict Dis 2016;35:58-65.

20. Snoek A, Levy N, Kennett J. Strong-willed but not successful: The importance of strategies in recovery from addiction. Addict Behav Rep 2016;4:102-7.

21. Grapevine AA. The twelve traditions. Alcoholics Anonymous ed, 1949.

22. Pedregosa F, Varoquaux G, Gramfort A, et al. Scikit-learn: machine learning in python. J Mach Learn Res 2011;12:2825-30.

23. Gainsbury SM. Review of self-exclusion from gambling venues as an intervention for problem gambling. J Gamb/ Stud 2014;30:229-51.

24. Fogarty M, Taylor-Rodgers E. Understanding the self-exclusion process in the act. Centre for gambling research at the australian national university, commissioned by the act gambling and racing commission, 2016.

25. Parke J, Parke A, Harris A, et al. Restricting Access: Self-Exclusion as a Gambling Harm Minimisation Measure in Great Britain. The Journal of Gambling Business and Economics 2014;8:52-92.

26. Art. 38 Mesures de prévention et détection précoce, Art. 41 Interdiction de jeu et exclusion, 2004. 935.521.21.

27. Tremblay N, Boutin C, Ladouceur R. Improved self-exclusion program: preliminary results. J Gamb/ Stud 2008;24:505-18.

28. Luquiens A, Tanguy ML, Lagadec M, et al. The Efficacy of Three Modalities of Internet-Based Psychotherapy for Non-TreatmentSeeking Online Problem Gamblers: A Randomized Controlled Trial. J Med Internet Res 2016;18:e36.

29. Nowatzki NR, Williams RJ, Williams RJ. Casino self-exclusion programmes: A review of the issues ${ }^{1}$. Int Gamb/ Stud 2002;2:3-25. 